presented and the implications for GC testing in our clinic population discussed.

\section{P25 INVESTIGATING FACTORS FOR INCREASED GONORRHOEA RE-INFECTION IN MSM ATTENDING A GU CLINIC: A QUALITATIVE STUDY}

'Lara Payne, 1,2 David Lawrence*, ${ }^{1,2}$ Suneeta Soni, 'Carrie Llewellyn, 'Gillian Dean. ${ }^{1}$ Brighton and Sussex Medical School, Brighton, UK; ${ }^{2}$ Brighton and Sussex University Hospitals NHS Trust, Brighton, UK

\subsection{6/sextrans-2015-052126.69}

Background/introduction In 2013, 63\% of gonorrhoea infections in England were in men who have sex with men (MSM), in whom the annual incidence increased by $26 \%$ (PHE). In our clinic, annual incidence increased by $28.8 \%$ (2013) and re-infection (a second infection within 1-year of initial infection) rose from $6.7 \%$ as a proportion of total infections (2009) to $19.4 \%$ (2013). This is concerning given increasing reports of antibiotic resistant gonorrhoea.

Aim(s)/objectives The aim of this study was to explore reasons for repeat gonorrhoea infections among MSM.

Methods We interviewed 16 MSM about knowledge of gonorrhoea, attitudes to safe sex and antibiotic resistance.

Results Mobile applications were used to meet casual sex partners and arrange impromptu group-sex parties with partner anonymity making contact tracing difficult. The use of recreational drugs was widespread and could result in unsafe sexual practices. Participants felt their behaviour was unlikely to change despite knowing there was increased gonorrhoea prevalence and frequently felt resigned to repeat infections. Participants thought global antibiotic resistance was concerning, but felt behaviour would change only if there was local evidence of this. It was highlighted that new technologies could increase awareness around local STI trends and services for those at risk.

Discussion/conclusion MSM's use of geosocial networking applications to arrange sex could also be harnessed to increase awareness and advertise testing opportunities. Enhanced interventions at initial diagnosis may also be beneficial. In some cases risk-taking behaviours are unlikely to change and for these men regular sexual health screens should be encouraged.

\section{P26 HOW VALUABLE IS LUMBAR PUNCTURE IN THE DIAGNOSIS OF NEUROSYPHILIS?}

Ruth Byrne*, Amy Dehn Lunn, Nneka Nwokolo. Chelsea and Westminster Hospital, London, UK

\subsection{6/sextrans-2015-052126.70}

Background/introduction UK syphilis incidence is rising. There are no national data on neurosyphilis prevalence. The CDC defines confirmed neurosyphilis as positive CSF VDRL at any syphilis stage and presumptive neurosyphilis as non-reactive CSF VDRL, raised CSF protein or WCC, positive serum VDRL and clinical symptoms/signs of neurosyphilis in the absence of any other causes. VDRL and RPR perform the same function; however, sensitivity of VDRL in CSF is poor $(30-70 \%)$ and RPR even poorer.

Aim(s)/objectives To identify and characterise patients referred and treated for neurosyphilis in a London HIV/GUM service.

Methods We reviewed all cases referred for investigation of possible neurosyphilis September 2012-September 2014.
Results 1615 new diagnoses of syphilis were identified. 34 were referred for suggestive symptoms. 24(71\%) were treated although only $6(25 \%)$ met CDC criteria for confirmed or presumptive neurosyphilis. Of those treated, $67 \%$ were HIV+, 4 had positive RPR ( 2 had no other CSF abnormality), 10 had positive TPPA only and 3 had no CSF abnormality.

Discussion/conclusion No single laboratory test is both sensitive and specific making diagnosis challenging. CSF interpretation may be particularly difficult in HIV+ individuals as HIV itself can cause pleocytosis and elevated protein concentrations. Conversely, Marra et al. showed that in 32\% of HIV+ patients with neurosyphilis, the only CSF abnormality was a positive VDRL. We suggest that given the poor sensitivity of CSF RPR, and that CSF may be normal in neurosyphilis, most decisions to treat for neurosyphilis should be based on clinical symptoms/ signs rather than CSF findings.

\section{P27 EXTRA-GENITAL CHLAMYDIA TESTING IN HETEROSEXUAL PATIENTS. IS IT WORTH IT?}

Laura Percy*, Kate Langley, Emily Harrison, Nathan Sankar, Laura Michell. New Croft Centre, Newcastle Upon Tyne, UK

\subsection{6/sextrans-2015-052126.71}

Background/introduction Current clinic policy is to offer extragenital testing to all patients reporting a history of active oral sex and/or receptive anal sex. These swabs are analysed using the Aptima Combo II platform for Chlamydia trachomatis (CT). $\operatorname{Aim}(\mathrm{s}) /$ objectives With analysis costing $£ 6.20$ per swab we sought to explore the cost effectiveness and review positive case with collateral contact information and symptoms history to support a positive diagnosis.

Methods Inclusion criteria were heterosexual patients with exclusively extra-genital CT who did not present as CT contact. We performed retrospective case note review of 63 sets of notes to determine symptom history, concurrent STI diagnosis and contact diagnosis.

Results Over the year, a total of 12076 throat swabs were sent in this group. There were 39 confirmed positive results giving swabs sent per positive result ratio of 310:1. Or a cost of $£ 1922$ per positive result. For rectal swabs; a total of 1156 were sent. There were 24 positive results giving swabs sent per positive result ratio of $48: 1$, or a cost of $£ 297.60$ per positive result. $5 \%$ of patients with a positive extra-genital swab result gave a history of throat or rectal symptoms. $4 \%$ had a concurrent STI diagnosis, $40 \%$ of those with traceable contacts had at least one positive contact.

Discussion/conclusion Routine extra-genital screening is costly but this review demonstrates its value for detection of individual cases which would have been missed. In addition the high proportion of positive contacts adds weight to the debate for extragenital testing of all contacts.

\section{P28 EXTRA-GENITAL GONORRHOEA TESTING IN HETEROSEXUAL PATIENTS. IS IT WORTH IT?}

Laura Percy*, Kate Langley, Emily Harrison, Nathan Sankar, Laura Mitchell. New Croft Centre, Newcastle Upon Tyne, UK

\subsection{6/sextrans-2015-052126.72}

Background/introduction Current clinic policy is to offer extragenital testing to all patients reporting a history of active oral 